\title{
Alteration of Amino Acid Profiling Influenced by the Active Ingredients of DanHong Injection After Prescription Optimization
}

This article was published in the following Dove Press journal:

Drug Design, Development and Therapy

\author{
Zhili Guo $\mathbb{D}^{\prime}$ \\ Yan Zhu ${ }^{2}$ \\ Wenjuan $\mathrm{Xu}^{3}$ \\ Kaitao Luo' \\ Hongbin $\mathrm{Xiao}^{4}$ \\ Zhong Wang ${ }^{3}$
}

'Chinese Medicine Department, Jiaxing Hospital of Traditional Chinese Medicine, jiaxing University, Jiaxing, Zhejiang, 314000, People's Republic of China; ${ }^{2}$ Chinese Medicine Department, Beijing Electric Power Hospital, Capital Medical University, Beijing 10073, People's Republic of China; ${ }^{3}$ Chinese Medicine Pharmacology, Institute of Basic Research in Clinical Medicine. China Academy of Chinese Medical Sciences, Beijing 100700, People's Republic of China; ${ }^{4}$ Beijing University of Chinese Medicine, Chinese Medicine Institute, Beijing I00029, People's Republic of China
Correspondence: Kaitao Luo; Zhong

Wang

Tel +86057382079269;

$+86|0640| 44 \mid I-3308$

Emaillkt7408I3@I63.com;

zhonw@sina.com
Introduction: The aim of this work was to optimize the formulation composition of DanHong injection and to study the disturbance of microscopic components of cerebral ischemia in amino acid metabolites and metabolic pathways. The subtle relationship among these three substances and the influence of metabolic pathways were also studied.

Methods: In this study, the central composite design (CCD) matrix and response surface methodology (RSM) were used to design the experiments and to evaluate the interactive effects of three substances. Targeted metabolomics was used to detect the amino acid variation in CCD sets. Results: Response surfaces were generated, and the formulation was optimized by superimposing the contour plots. It was found that the optimum values of the responses could be obtained at an SAB concentration (x1) of 8-9 mg/kg, a TSN concentration (x2) of 14-16 mg/kg, and an HSYA yellow A concentration (x3) of $6 \mathrm{mg} / \mathrm{kg}$. Statistical analysis showed that the three independent variables had significant effects $(p<0.05)$ on the responses. A total of 22 experimental runs were performed, and the kinetic data were analyzed using a second-order polynomial. Model algorithm calculation indicated that glutamic acid, serine, leucine, glycine, and valine had a very close correlation with the active ingredients. Methionine, aspartic acid, asparagine, glutamic acid, and valine were important for distinguishing different groups, and they were identified as potential biomarkers. Cluster analysis and pathway analysis indicated that the valine, leucine, and isoleucine degradation (VLI degradation) pathway was the major metabolic pathway. Arginine and proline metabolites were most frequently detected, and they were closely associated with other networks according to the network analysis results. VLI degradation pathway and arginine and proline metabolism pathway had a significant influence on cerebral ischemia.

Discussion: The integration of CCD and metabolomics may be an effective strategy for optimizing the formulation composition and identifying the mechanism of action of traditional chinese medicine.

Keywords: amino acid metabolomics, central composite design, metabolic pathways, cerebral ischemia, DanHong injection, HPLC-FLD test, traditional Chinese medicine

\section{Introduction}

The physical properties of all substances are determined by their main components and microstructures; therefore, it is important to ensure the stable uniformity of these components of Chinese medicinal materials. Identifying the relationship between the characteristics of the main components and their pharmacologic effects is a critical problem in the modernization of Traditional Chinese Medicine (TCM). ${ }^{1-3}$ The central composite design matrix and response surface methodology (CCD-RSM) is an approach that has been widely applied to the formulation and process optimization in pharmaceutics. ${ }^{4-6}$ As there 
are a number of variables involved in the development of a pharmaceutical formulation and its associated process, the advantages of a systematic approach using experimental design optimization techniques and chemometric data evaluation are obvious. ${ }^{7-11}$

DanHong (DH) injection is a traditional Chinese medicine widely used for treating cerebral ischemia by promoting blood circulation, dilating blood vessels, lowering levels of fat in the blood, exerting an anti-inflammatory effect in traditional medicine. It is commonly used in clinical practice in China for treating conditions such as cerebral infarction, thrombosis, trauma, subarachnoid hemorrhage, subdural hematoma, and the recurrence of fundus or retinal hemorrhage. ${ }^{12}$ The highperformance liquid chromatography (HPLC) fingerprint from different numbers of DH injection and the quality control condition of DH injection can be seen in Figures S1 and $\underline{\mathrm{S} 2}$. The substances tested were Salvia miltiorrhiza and safflower, with main active ingredients including salvianolic acid $\mathrm{B}$ (SAB), tanshinol (TSN), and hydroxysafflor yellow pigment A (HSYA). The main reason for selecting these ingredients as the research objects is that they are the main components and the typical representatives of DH injection. ${ }^{13-15}$ Research has shown that SAB, TSN, and HSYA may have a positive effect on brain damage. Chen Yulin et $\mathrm{al}^{16}$ explored the protective effects of the active ingredients of Salvia miltiorrhiza and safflower (TSN, SAB, HYSA, and salvianolic acid A (SAA)) in different combinations on cerebral ischemiareperfusion injury in rats, and found that the active components can play a protective role in endoplasmic reticulum stress and inflammation in these rats. Song Jinjun et $\mathrm{a}^{17}$ also found that the water-soluble components of Salvia miltiorrhiza and safflower may significantly reduce the symptoms of neurological deficits, promote the reversal of symptoms of neurological deficits, visibly decrease the volume of brain tissue infarction, reduce brain index and brain water, increase the activity of superoxide dismutase (SOD), reduce the levels of malondialdehyde (MDA), IL-1 $\beta$, IL- 6 , and TNF- $\alpha$, and inhibit reduction or disappearance of Nissl bodies.

It has been shown that the development of cerebral ischemia is closely related to the metabolic spectral migration of amino acids. ${ }^{18}$ Amino acid analysis is an important part of metabolomics, ${ }^{19}$ and a previous study suggested that Chinese medicine could treat rats suffering from stroke-related ischemia/reperfusion (I/R) injury by ameliorating the disorder in amino acid metabolism. ${ }^{20}$ Therefore, our study was conducted to explore how the relative contents of amino acids and their metabolic pathways changed after the alteration of amino acid profiles by the active ingredients of $\mathrm{DH}$ injection.
We then studied the disturbance of the microscopic components of cerebral ischemia in amino acid metabolites and metabolic pathways to investigate via metabolomics whether the amino acid metabolome is affected on the microscopic level after prescription optimization. Metabolomics, which focuses on the relative relationship between metabolites and physiological and pathological changes, was used to further assess metabolic features after the introduction of three active ingredients to determine the potential benefit in treating cerebral ischemia, and to identify the relationship between the active ingredients and their metabolites. ${ }^{21-24}$ This may help to identify the mechanism of action of TCM.

In our study, the chemometric method was used for the screening and optimization of factors influencing the extraction efficiency by employing CCD-RSM. The purpose of our work is to illustrate the suitability of this approach for selecting an optimal formulation and analyze the relationship between the optimized dose with microscopic amino acid metabolites and pathways.

\section{Materials and Methods Central Composite Design}

CCD has been widely used for building a second-order model, and it requires a minimal number of experiments. The independent process variables were obtained at an $\mathrm{SAB}$ concentration (x1), a TSN concentration ( $\mathrm{x} 2$ ), and an HSYA concentration (x3), which were defined as the factors, and the volume of cerebral infarction (y) was used as the response. The data obtained for the three responses in each trial were fitted to the classical second-order polynomial model. These variables were investigated at five levels $(-1.732,-1,0,1$, and 1.732), and 20 batches were prepared and evaluated for the volume of cerebral ischemia $(y)$ as the dependent variable. Second-order models were obtained to fully describe the influence of the independent variables on the selected responses.

The Minitab software (V17.2) package was used to design and evaluate the response of these three independent variables at five levels. The ranges for the selected levels of the four variables are shown in Table 1. The experimental extraction yield at different selected levels of variables is shown in Table 2 for 20 batches.

$$
\begin{aligned}
y= & b 0+b 1 \times 1+b 2 \times 2+b 3 \times 3+b 4 \times 21+b 5 \times 2 \\
& +b 6 \times 23+b 7 \times 1 \times 2+b 8 \times 1 \times 3+b 9 \times 2 \times 3
\end{aligned}
$$

The formulations are listed in Table 1 in a coded form. The experiments were conducted in a random sequence 
Table I Factors and Levels of the Circumscribed Central Composite Design

\begin{tabular}{|l|l|l|l|l|l|}
\hline \multirow{2}{*}{ Factors } & \multicolumn{4}{|l|}{ Levels } \\
\cline { 2 - 6 } & -1.732 & -1 & $\mathbf{0}$ & $\mathbf{I}$ & $\mathbf{I . 7 3 2}$ \\
\hline Tanshinol (XI) & 1.5 & 13.86 & 30.75 & 47.72 & 60 \\
Salvia acid B (X2) & $\mathrm{I}$ & 13.86 & 30.75 & 47.72 & 60 \\
Hydroxysafflor yellow & 0.2 & 2.67 & 6.1 & 9.52 & 12 \\
A (X3) & & & & & \\
\hline
\end{tabular}

with the center points being repeated three times to minimize experimental error.

The materials and methods of sample acquisition and preprocessing, preparation of the middle cerebral artery occlusion model, calculation of cerebral ischemia volume (in Figure S3), HPLC sample treatment, instruments and reagents, online derivatization, metabolic profiling, validation of assay method, and data and pathways analysis were the same as in the references, ${ }^{25}$ and were placed on the attachment. (See Supplementary Material)

\section{Results}

\section{Analyses of Response Surfaces and Fitted Data}

We primarily investigated the active components of the $\mathrm{DH}$ injection. The preparation of prescription DH injection was performed on the basis of Table 2 . The results showed that 20 different prescriptions had different effects on the treatment of cerebral infarction in rats, and the area of cerebral infarction was an important index in the design of the star point.

The quadratic polynomial model for describing the mathematical relationship between the inspection of indexes and the three factors was ascertained based on the concentrations of SAB, TSN, and HYSA, with the index of cerebral infarction as the area of investigation, using the multivariate linear model, and then the response surface and contour map were drawn to determine the best prescription. A reliable quantitative relationship between the three factors and the index was obtained through the quadratic polynomial model. The predicted values of the optimized prescription were very close to the measured values. According to the polynomial equation obtained by the model, the $3 \mathrm{D}$ response surface and the 2D contour surface of each index and factor were plotted by Matlab software (V17.2).

It can be seen from the response surface chart that as the area of " 1 cerebral infarction \%" gradually decreased, the dose of HSYA increased monotonically (Figure 1A), while the doses of TSN and SAB were increased initially and then decreased (Figure 1B and C). The optimal prescription for the injection obtained from the composite contour curves was TSN $14-16 \mathrm{mg} / \mathrm{kg}$, SAB $8-9 \mathrm{mg} / \mathrm{kg}$, and HSYA $6 \mathrm{mg} / \mathrm{kg}$. The response surface was evaluated and optimized based on the relationship between the cerebral infarction area and the composition of three ingredients, and then the model was obtained. The following second-order polynomial equation was proposed for the prediction of CI yield as a function of different variables:

$$
\begin{aligned}
\mathrm{y}= & 1-(0.4982+0.0547 * \mathrm{x} 1+0.0601 \\
& * \mathrm{x} 2-0.000236 * \mathrm{x} 1^{2}-0.0029 * \mathrm{x} 2^{2} \\
& +0.0012 * \mathrm{x} 1 * \mathrm{x} 2)-0.0070 * \mathrm{x} 2 \\
& * \mathrm{x} 3, \operatorname{adj} \mathrm{R}=0.494, \mathrm{p}=0.0158 \\
& \text { Press }=0.058, \mathrm{R}=0.65, \mathrm{BIC} \\
& =-13.7331
\end{aligned}
$$

\section{Screening of the Relationship Between the Three Active Components of $\mathrm{DH}$ Injection with the 20 Variables of the CCD Group}

We next studied the disturbance of the microscopic components of cerebral ischemia in amino acid metabolites and metabolic pathways. Metabolomics was used in this study to identify the relationship between the active ingredients and their metabolites. Typical HPLC-FLD amino acid profiling is shown in Figure 2A. The potential metabolites were identified by comparing corresponding standards according to their retention times. In all, the following order of 20 metabolites was analyzed: 1) aspartic acid, 2) glutamic acid, 3) asparagine, 4) serine, 5) histidine, 6) glycine, 7) alanine, 8) citrulline, 9) threonine, 10) arginine, 11) taurine, 12) tyrosine, 13) valine, 14) methionine, 15) tryptophan, 16) phenylalanine, 17) isoleucine, 18) ornithine, 19) leucine, and 20) lysine (norvaline is the internal standard [IS]). The relationship between the 20 variables of the active ingredient compatibility group (CCD group) and the compound monomer was screened. We arranged the doses of the compound monomer from small to large, and they were grouped on the basis of the concentration. Obviously, the contents of glutamic acid and valine changed according to the concentrations of the three compounds. The activity of TSN was closely associated with glutamic acid and valine. The activity of HSYA was closely 
Table 2 The Preparation of Prescription DH Injection

\begin{tabular}{|c|c|c|c|c|c|}
\hline Groups & $\mathbf{N}$ & Tanshinol & Salvia Acid B & Hydroxysafflor Yellow A & Cerebral Ischemia \\
\hline I & 6 & 6.27 & 3.11 & 1.5 & $27.80 \pm 5.79$ \\
\hline 2 & 3 & 6.27 & 9.65 & 1.5 & $23.44 \pm 8.72$ \\
\hline 3 & 3 & 19.45 & 9.65 & 1.5 & $29.15 \pm 5.49$ \\
\hline 4 & 3 & 19.45 & 3.11 & 1.8 & $18.30 \pm 6.18$ \\
\hline 5 & 3 & 25 & 6.51 & 3.15 & $27.73 \pm 5.68$ \\
\hline 6 & 4 & 13.125 & 12.4 & 3.15 & $27.94 \pm 10.10$ \\
\hline 7 & 4 & 13.125 & 6.51 & 0.3 & $27.15 \pm 7.24$ \\
\hline 8 & 6 & 13.125 & 6.51 & 6 & $27.43 \pm 8.40$ \\
\hline 9 & 12 & 13.125 & 6.51 & 3.15 & $21.20 \pm 6.09$ \\
\hline 10 & 6 & 47.72 & 13.47 & 2.69 & $23.32 \pm 8.40$ \\
\hline II & 3 & 13.86 & 47.61 & 2.69 & $28.60 \pm 7.48$ \\
\hline 12 & 4 & 47.72 & 47.61 & 2.69 & $27.40 \pm 7.00$ \\
\hline 13 & 3 & 13.86 & 13.47 & 9.52 & $20.46 \pm 1.32$ \\
\hline 14 & 3 & 1.5 & 30.5 & 6.1 & $21.31 \pm 8.48$ \\
\hline 15 & 3 & 60 & 30.5 & 6.1 & $18.33 \pm 5.96$ \\
\hline 16 & 3 & 30.75 & I & 6.1 & $|8.5| \pm 0.65$ \\
\hline 17 & 3 & 30.75 & 30.5 & 12 & $26.30 \pm 2.28$ \\
\hline 18 & 3 & 30.75 & 30.5 & 12 & $26.30 \pm 2.28$ \\
\hline 19 & 3 & 30.75 & 30.5 & 12 & $26.30 \pm 2.28$ \\
\hline 20 & 3 & 30.75 & 30.5 & 12 & $26.30 \pm 2.28$ \\
\hline Vehicle & 6 & & & & $35.17 \pm 8.32$ \\
\hline Sham & 9 & & & & 0 \\
\hline Total & 87 & & & & $21.95 \pm 11.23$ \\
\hline
\end{tabular}

associated with leucine (Figure 2B). The relationship between the area of cerebral infarction and amino acid concentration was then further analyzed. The box was the combination of cerebral infarction and amino acid concentration according to the compound (Figure 2C). Cerebral infarction was transformed into " 1 cerebral infarction \%"
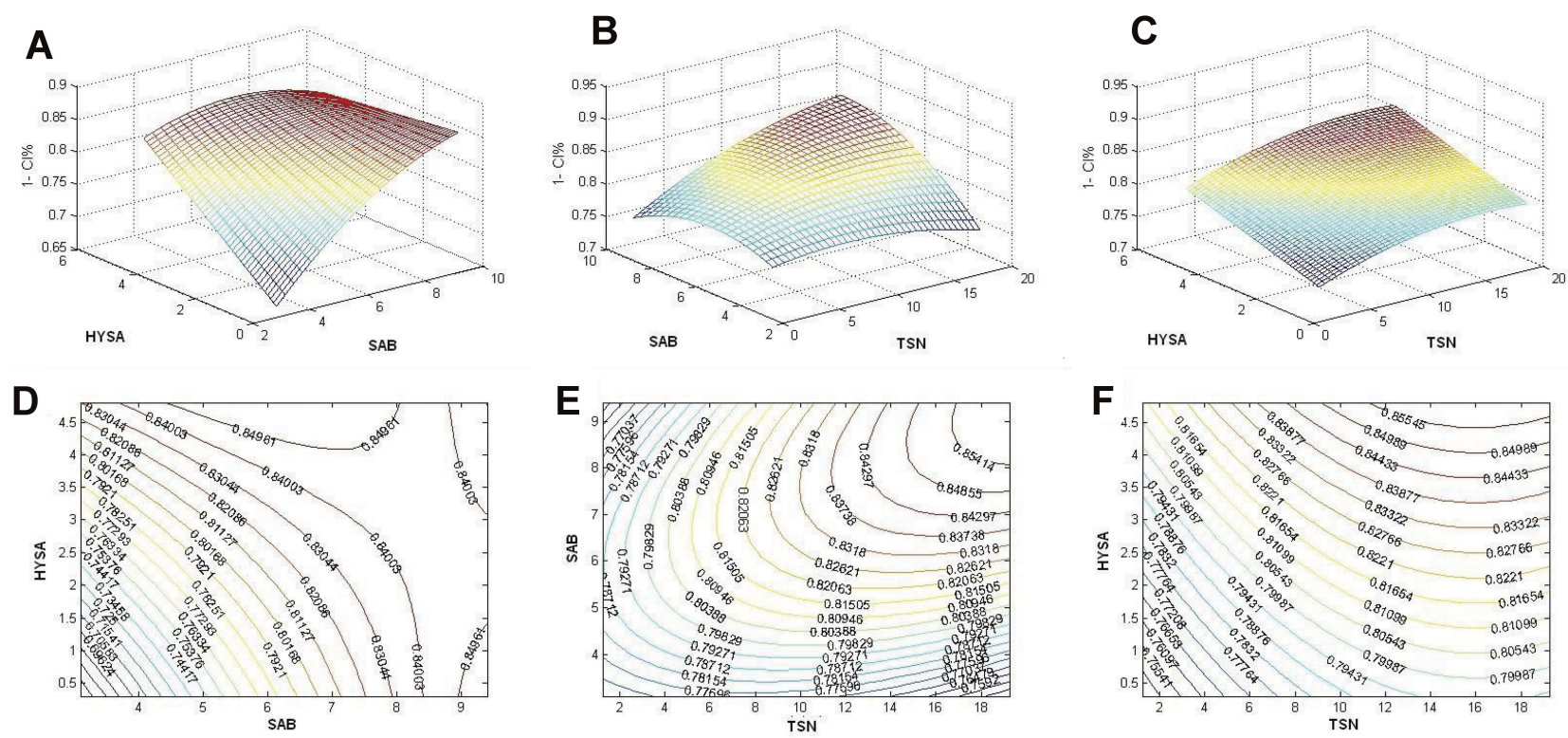

Figure I Response surface model (RSM) showing the influence of the dose of active component concentration on I-cerebral infarction \%. (A-C) The area of "I-cerebral infarction \%" gradually decreases (just the increase of cerebral infarction \%) in the effect surface chart. X-axis represents the doses of TSN and SAB and HYSA; Y-axis representsI-cerebral infarction \%. The doses of TSN and SAB were all increased at first and then decreased, and the dose of HSYA increased monotonically. (D-F) Predicted 2D contour maps illustrating the effect of $X 1, X 2, X 3$ on the concentration of $Y 1$. 

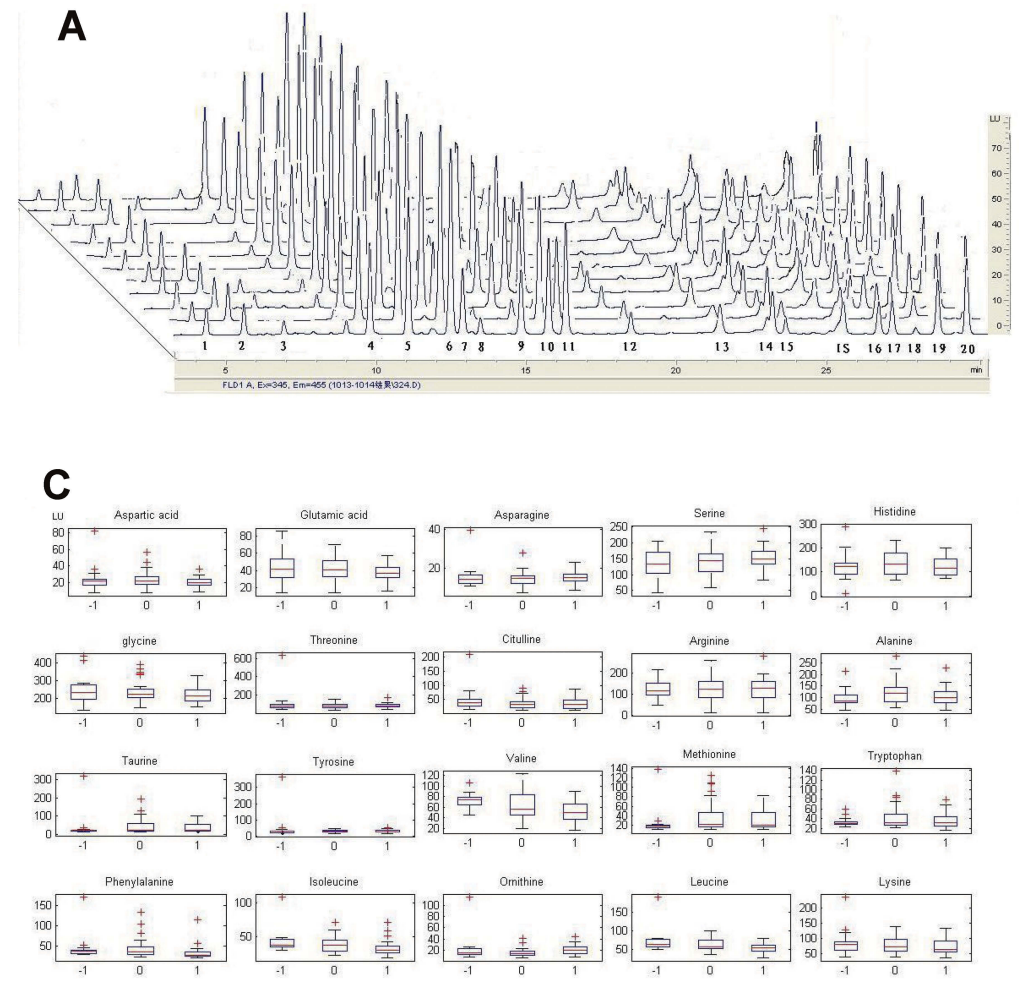

B
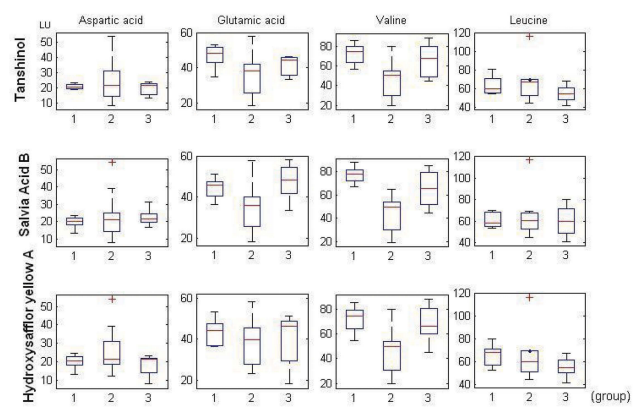

D

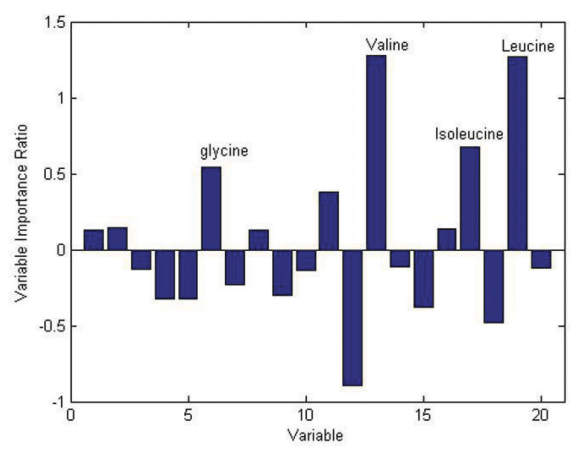

Figure 2 Screening of the relationship between the three active components of DH injection with the 20 variables of the CCD group. (A) Twenty kinds of amino acids of the samples in the 3 groups were separated in 30 mins completely; $X$-axis represents retention time, and Y-axis represents peak intensity; (B) The relationship between the 20 variables of the active ingredient compatibility group (CCD group) and the doses of the compound monomer from small to large, and they aregrouped by concentration. (C). Analysis of the relationship between cerebral infarction area and amino acid concentration; the box was the combination of cerebral infarction and amino acid concentration by compound. (D) Model group analysis method was used to evaluate the standardized effects of variables. The ordinate represents the importance of the reaction variables.

and then it was allocated into the following three groups: -1 (0.51-0.7), 0 (0.7-0.9), and 1 (0.9-1). Figure 2B shows that with the monotonic increase in the CI area, Glu, Ser, Leu, Gly, Val, and the CI area showed a monotonically increasing or decreasing trend, especially Val. In this case, variance analysis demonstrated the significance of the concentration of Val, which showed a negative effect on the infarct area. Thus, with an increasing presence of Val, a smaller CI volume was observed. Evaluation of the standardized effects of the variables in the CCD group was also performed. The model group analysis method was used to evaluate the standardized effects of the variables. The ordinate represents the importance of the reaction variables (Figure 2D); glycine, valine, isoleucine, and leucine showed an impact in distinguishing the 20 prescription groups.

\section{Multivariate Statistical Analysis of the Amino Acid Metabolites of the 22 Groups}

We also used chemometrics to analyze the changes in amino acid metabolite microcomposition. PCA (principal component analysis) is a multivariate statistical analysis method that uses linear transformation to select a few important variables. Initially, 2D- and 3D-PCA score plots were obtained from the HPLC-FLD data of the three groups (Figure 3A and B), using PCA analysis. A nearly complete separation in metabolic profiling was detected. The $\mathrm{X}$-axis represents the score of principal component 1 ( $\mathrm{PC} 1)$, while the $\mathrm{Y}$-axis indicates the score of PC2 (R2X=0.845; Q2=0.516) (Figure 3B).

Outlying trend analysis showed that in the sample space, the points that were inconsistent with the general behavior and characteristics of other samples became the outliers, as shown in Figure 3C. Only five potential outlier variables obviously deviated. Next, 15 variables were added in these treatment groups, which were screened out based on the variable importance of projection (VIP) value $>1$ compared with the vehicle group (Figure 3D). Features were ranked according to their contributions to the classification accuracy (mean decrease accuracy). Methionine, aspartic acid, asparagine, glutamic acid, valine, etc., were excreted when the VIP value was $>1$, and Heatmap and Hierarchical 
A

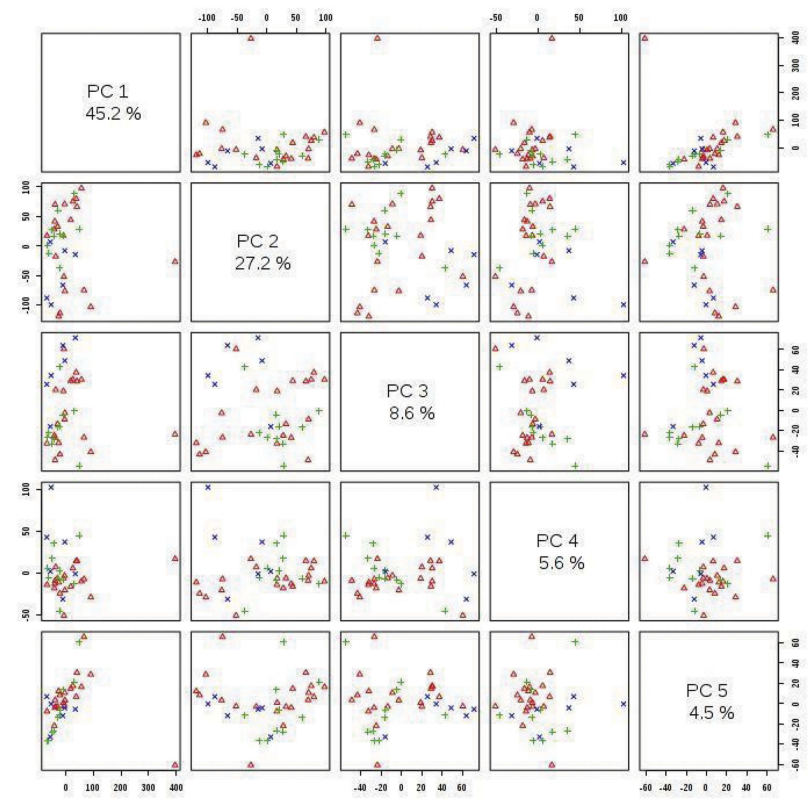

C

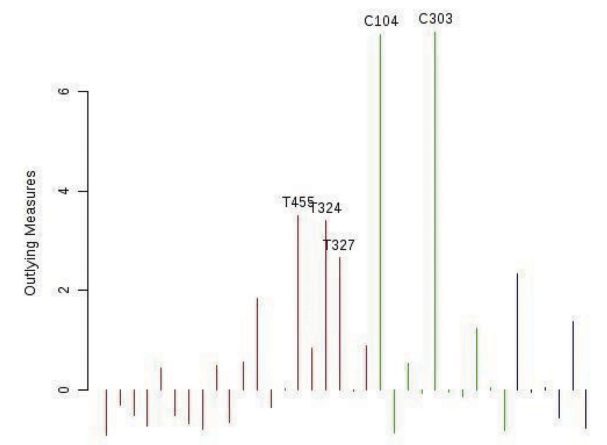

Samples

$\mathbf{E}$

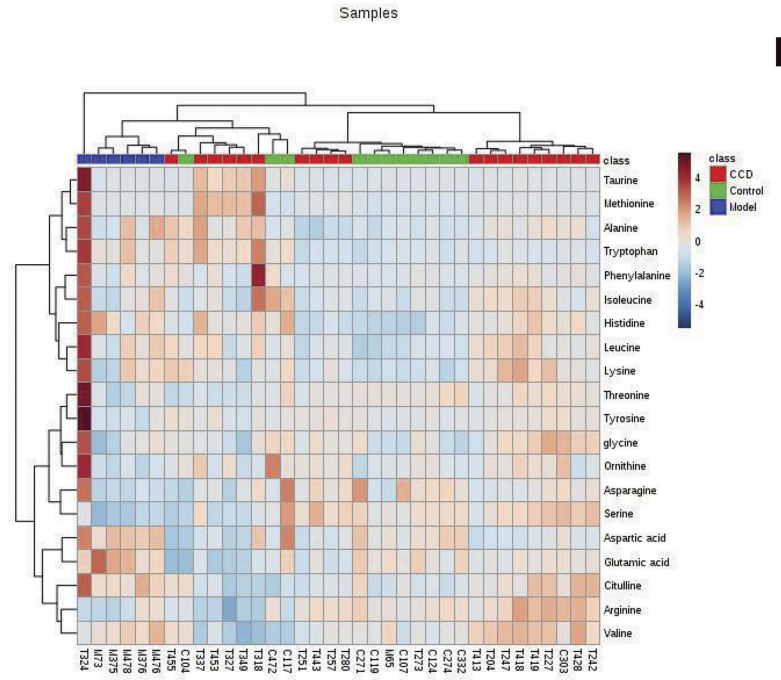

B
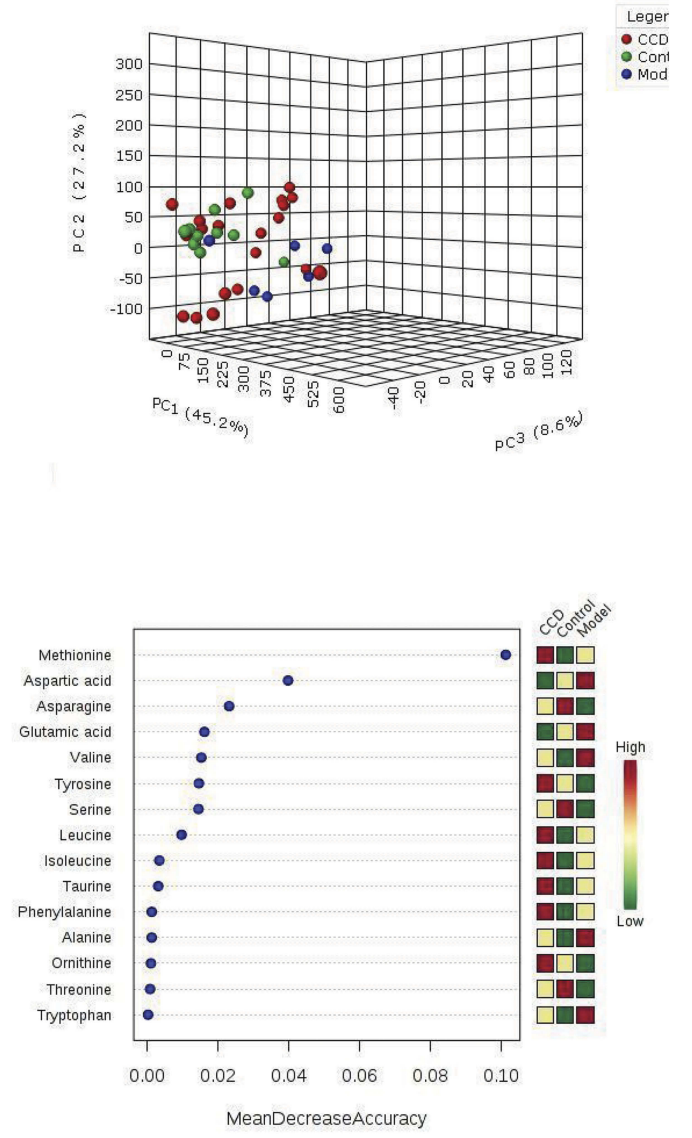

F

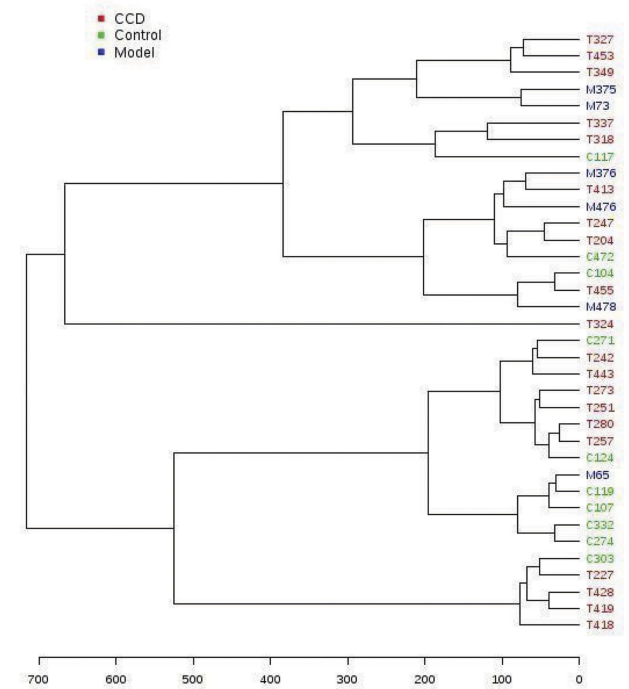

Figure 3 Multivariate statistical analysis of the amino acid metabolites. (A, B) 2D- and 3D-PCA score plots were initially obtained from the HPLC-FLD data of the 3 groups PCA score plots. (C) Five potential outliers are departure obviously with the general. (D) Fifteen variables which were screened out based on a variable importance of projection (VIP) value $>0$ compared with the vehicle group. (E, F). We use heatmap and hierarchical clustering to identify samples/features that are unusually high/low. 
Clustering were used to identify the samples/features that were unusually high or low (Figure $3 \mathrm{E}$ and $\mathrm{F}$ ). The two common findings were that the three groups could be distinguished by amino acid detection in space by cluster analysis, and that the CCD group was more likely to resemble the healthy group.

\section{Effect of Doses Changed After CCD Design on Serum Amino Acid Metabolic Pathways}

The detailed results of the pathway analysis and the changing processes in amino acid metabolic pathways compared with those in the vehicle and sham groups are illustrated in Table S2. When comparing the vehicle group with the sham group, biomarkers were obtained without interference, which can be further researched on biological significance. When comparing from vehicle group with the CCD (1-20) group, some biomarkers regulated by efficient active ingredients were obtained.

The changing processes of the amino acid metabolic pathways were compared with those in the vehicle and sham groups. In $\mathrm{CCD}$, the amino acid metabolic pathways in $20 \mathrm{CCD}$ groups were concentrated in a total of 78 channels
(IF $>0.1$, and nine non-overlapping metabolic pathways were involved. The frequency of these can be seen in Figure 4A (see the attached Table $\mathrm{S} 1$ for the full form of the abbreviation). We found that Arginine and proline metabolism (AAP metabolism) most commonly occurred during our research.

The detailed results from the pathway analysis are shown in Table S2, and the changing processes of amino acid metabolic pathways compared with the vehicle and sham groups are illustrated in Figure 4. The channel number and the properties of the pathways, such as the name of the related pathways, the total compound of the pathway, the number of hits, and the impact value, were all changed abnormally due to drug intervention according to MetPa and MetaboAnalyst; however, the relationship between this change law and CCD design cannot be analyzed.

The following potential biomarkers were found in the results: glutamic acid, serine, leucine, glycine, methionine, aspartic acid, asparagine, glutamic acid, and valine. These biomarkers were identified by MetPa and MetaboAnalyst systems, which can combine strong channel analysis with topological analysis to determine the most likely correlation pathways under experimental conditions. Nine nonoverlapping metabolic pathways were involved, and the
A

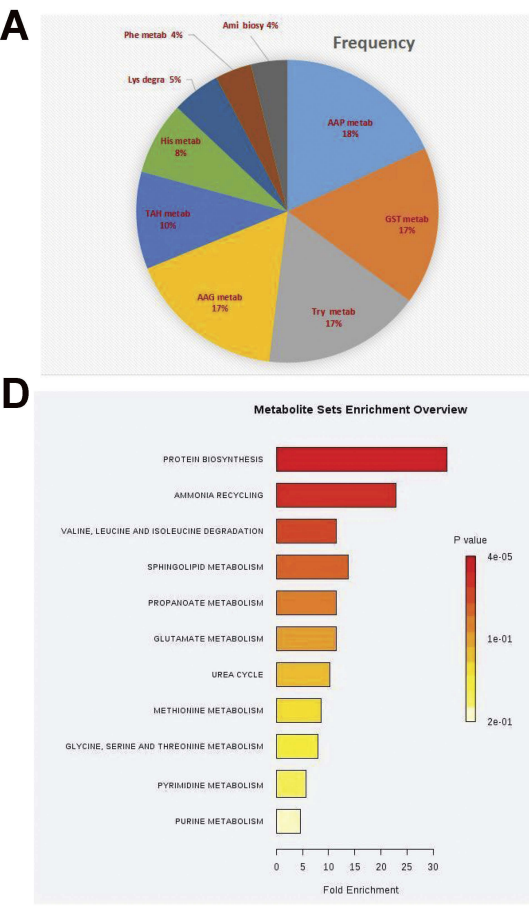

B

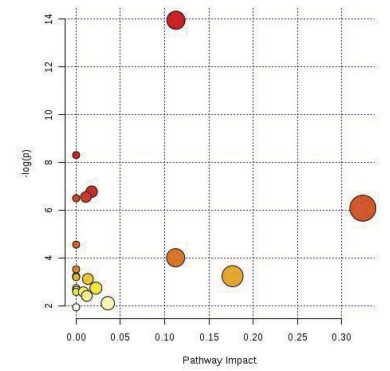

E

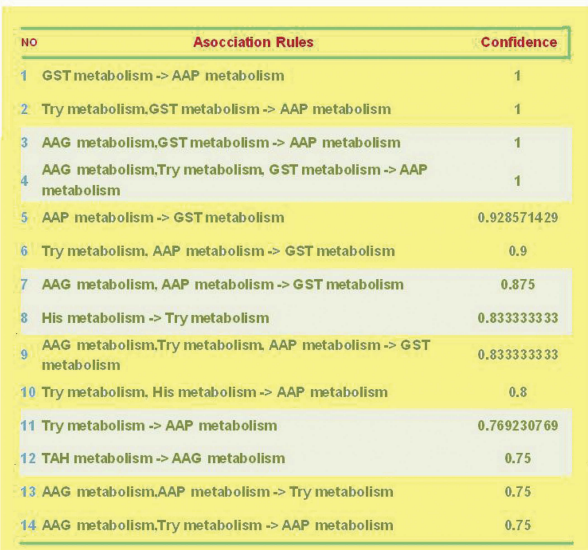

C

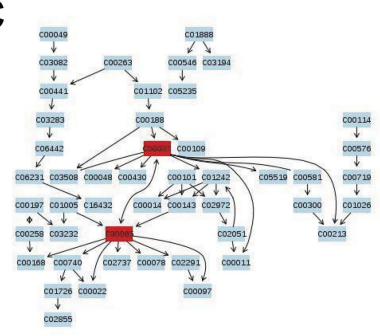

$\mathbf{F}$

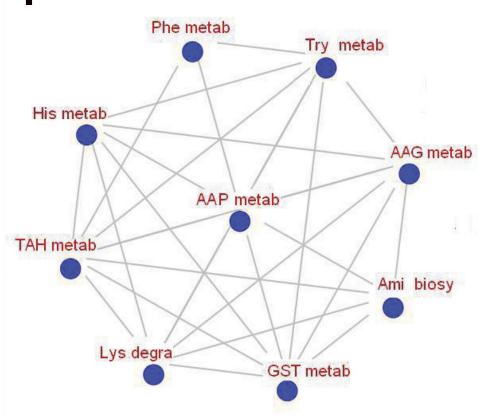

Figure 4 Effect of different doses of $\mathrm{DH}$ injection on serum amino acid metabolic pathways. (A) Nine non-overlapping metabolic pathways were involved and the frequency of them can be seen. (B) The graph of the series of concentrated metabolic pathways is obtained; the maximal metabolic pathway was VLI degradation. (C, D) The analysis of the centralized effect of the 78 channels also showed that VLI degradation held third place falling behind protein biosynthesis and ammonia recycling. (E, F) The result of association rule was shown and the network display of amino acid metabolic pathways. 
frequency of these can be seen in Figure 4A. The graph of the series of concentrated metabolic pathways was obtained (Figure 4B), and the main metabolic pathway was the VLI degradation pathway (Figure 4C). The analysis of the concentrated effect of the 78 channels also showed that the VLI degradation pathway occupied the third place, following protein biosynthesis and ammonia recycling (Figure 4D). The result of the association rule is shown in Figure 4E, and the network display of amino acid metabolic pathways is shown in Figure 4F. The confidence level of the rules was greater than 0.7. AAP metabolism had the highest correlation with the other pathways, as the confidence level was equal to 1 .

Based on the information above, we speculated that VLI degradation and AAP metabolism showed maximum changes during the metabolic process of cerebral infarction.

\section{Discussion}

The CCD response surface method was performed, and a good CCD model experiment that concluded the projection of the highest point and the metering was performed. Finally, the prescription optimization of DH injection was implemented based on the quadratic polynomial model for optimization. Then, we applied amino acid metabolomics to detect the plasma levels in 81 experimental rats and obtained a clear map of amino acid metabolism.

\section{Analysis of Various Signals and Metabolic Pathways of Cerebral Ischemic Disease}

We used the model algorithm to show that glutamic acid, serine, leucine, glycine, and valine were very closely correlated with the active ingredients. During chemometric analysis, we found that the amino acid metabolites actually caused a difference among the three groups.

Five variables (methionine, aspartic acid, asparagine, glutamic acid, and valine) were found to be important for distinguishing between the different groups. These were the potential biomarkers. Finally, on cluster analysis and pathway analysis, the potential biomarkers were found to be concentrated in the VLI degradation pathway. Arginine and proline metabolites were most frequently detected, and they were closely associated with other networks according to the analysis of network association rules. We speculated that VLI degradation and AAP metabolism showed maximum changes during the metabolic process of cerebral infarction. This experiment revealed the microcosmic effect of the optimized prescription of DH injection.
Through the analysis of network topology, nine biomarkers that significantly contributed to the classification of model groups and blank groups were preliminarily screened and identified. Two metabolic pathways were identified by the ischemic organization. The endogenous metabolites were mainly involved in protein biosynthesis, ammonia process, and VLI degradation.

In addition, the CCD group was closer to the blank group through the relative distance analysis of the blank group. The content of the metabolic product may recover to the level of healthy gradually.

\section{The Complex Relationship Between Amino Acid Metabolites and Cerebral Ischemic Diseases}

When there is valine deficiency, the function of the central nervous system in rats can be disturbed, and the limbs tremble in the form of ataxia. Excessive glutamate release and consequent calcium influx are associated with the death of ischemic neurons. The activity of TSN was closely associated with GLU/VAL.

Some studies have shown that TSN can reduce the damage to cerebral cortex cells in rats. ${ }^{26,27}$ The preliminary findings of metabolomic analysis imply a further recognition of the protective effects of cerebral ischemia on the endogenous material level by raising the valine level and decreasing the glutamate level. ${ }^{28,29}$

Also, arginine and proline metabolism, and valine, leucine, and isoleucine degradation were found in this section. The two signaling pathways associated with cerebral ischemia occupied a core and important position, which has an important influence on the generation and maintenance of cerebral ischemia. ${ }^{30,31}$

\section{Author Contributions}

All authors contributed to data analysis, drafting or revising the article, gave final approval of the version to be published, and agree to be accountable for all aspects of the work.

\section{Funding}

This article is supported by the research fund (2016BY 28045) and (GZS2017024).

\section{Disclosure}

The authors report no conflicts of interest in this work. 


\section{References}

1. Gfeller D, Butty F, Wierzbicka M. The multiple-specificity landscape of modular peptide recognition domains. Mol Syst Biol. 2011;7:484. doi: $10.1038 / \mathrm{msb} .2011 .18$

2. Gordon KE, Silverstein FS. Effects of perinatal stroke on striatal amino acid efflux in rats studied within vivo microdialysis. Stroke. 1991;22:928-932.

3. Carnicer M, Ten Pierick A, van Dam J. Quantitative metabolomics analysis of amino acid metabolism in recombinant pichiapastoris under different oxygen availability conditions. Microb Cell Fact. 2012;11:83. doi:10.1186/1475-2859-11-83

4. Jung JY, Lee H-S. H-NMR-based metabolomics study of cerebral infarction. Stroke. 2011;42:1282-1288. doi:10.1161/STROKEAHA. 110.598789

5. Chang RK, Guo X, Burnside BA, Cough RA. Fast dissolving tablets. Pharm Tech. 2000;24:52-58.

6. Dobetti L. Fast-melting tablets: developments and technologies. Pharma Tech. 2001;(Suppl):44-50.

7. Kuchekar BS, Arumugam V. Fast dissolving tablets. Indian J Pharm Educ. 2001;35:150-152.

8. Bradoo R, Shahani S, Deewan B, Sudarshan S. Fast dissolving drug delivery system. J Am Med Assoc India. 2001;4:27-31.

9. Vondrak B, Barnhart S. Dissolvable films for flexible product format in drug delivery. Pharma Technol Suppl. 2008;6:20-28.

10. Wu G, Bazer FW, Burghardt RC, et al. Proline and hydroxyproline metabolism: implications for animal and human nutrition. Amino Acids. 2011;40(4):1053-1063. doi:10.1007/s00726-010-0715-z

11. Andres RH, Ducray AD, Schlattner U, Wallimann T, Widmer HR. Functions and effects of creatine in the central nervous system. Brain Res Bull. 2008;76:329-343. doi:10.1016/j.brainresbull.2008.02.035

12. Wang S, Hong L. Clinical research progress of Danhong injection. Chi J Pract Nerv Dis. 2018;21(17):1862-1866.

13. Wang S. The Protective Effect of Danhong Injection on Cerebral Hemorrhage in Rats and Its Mechanism. Zhengzhou University; 2018.

14. Gutteridge JM, Smith A. Antioxidant protection by haemopexin of haem-stimulated lipid peroxidation. Biochem J. 1988;256(3):861. doi: $10.1042 / \mathrm{bj} 2560861$

15. Cai Y, Cho GS, Ju C, et al. Activated microglia are less vulnerable to hemin toxicity due to nitric oxide-dependent inhibition of JNK and p38 MAPK activation. $J$ Immunol. 2011;187(3):1314-1321. doi:10.4049/jimmunol.1002925

16. Yulin C, Haitong W, Huifen Z, et al. The protective effect of Dan in the effective distribution of safflower on cerebral ischemia-reperfusion injury in rats. 2018;49(16):3875-3881.

17. Jinjun S, Zhuchen Z, Bing C, Yuyan Z, Guojun W, Jin H. Study on the role of Dan in the water-soluble distribution of safflower against cerebral ischemia-reperfusion injury in rats. Chin J Tradit Chin Med. 2019;37(07):1616-1619.
18. Cheung BM, Li C. Diabetes and hypertension: is there a common metabolic pathway? Curr Atheroscler Rep. 2012;14:160-166. doi:10.1007/s11883-012-0227-2

19. Mäkinen VP, Soininen P, Forsblom C. 1H-NMR metabolomics approach to the disease continuum of diabetic complications and premature death. Mol Syst Biol. 2008;4:167-179. doi:10.1038/msb4 100205

20. Wang PR, Wang JS, Yang MH, Kong LY. Neuroprotective effects of Huang-Lian-Jie-Du-Decoction on ischemic stroke rats revealed by (1) H NMR metabolomics approach. J Pharm Biomed Anal. 2014;88:106-116. doi:10.1016/j.jpba.2013.08.025

21. Banecka-Majkutewicz Z, Sawuta W, Kadzinski L. Homocysteine, heat shock proteins, genistein and vitamins in ischemic stroke-pathogenic and therapeutic implications. Acta Biochim Pol. 2012;59: 495-499.

22. Bao Y, Zhao T, Wang X, Qiu Y, Su M, Jia W. Metabonomic variations in the drug-treated type 2 diabetes mellitus patients and healthy volunteers. J Proteome Res. 2009;8:1623-1630. doi:10.1021/pr800643w

23. Barber MN, Risis S, Yang C, et al. Plasma lysophosphatidylcholine levels are reduced in obesity and type 2 diabetes. PLoS One. 2012;7: e41456. doi:10.1371/journal.pone. 0041456

24. Bang HO, Dyerberg J, Sinclair HM. The composition of the Eskimo food in north-western Greenland. Am J Clin Nutr. 1980;33: 2657-2661. doi:10.1093/ajcn/33.12.2657

25. Guo Z-L, Zhu Y, Su X-T, et al. DanHong injection dose-dependently varies amino acid metabolites and metabolic pathways in the treatment of rats with cerebral ischemia. Acta Pharmacol Sin;2015. 748-757. doi:10.1038/aps.2014.167

26. Bourrinet P, Quevauviller A. Prosopinine, an alkaloid from Prosopis africana (Legumineous). Its effects on the central and autonomic nervous systems. C R Seances Soc Biol Fil. 1968;162:1138-1140.

27. Brouns R, Wauters A, Van D, et al. Decrease in uric acid in acute ischemic stroke correlates with stroke severity, evolution and outcome. Clin Chem Lab Med. 2010;48:383-390. doi:10.1515/ CCLM.2010.065

28. Cameron R, Savita K. Significance of brain tissue oxygenation and the arachidonic acid cascade in stroke. Antioxid Redox Signal. 2011;14:1889-1903. doi:10.1089/ars.2010.3474

29. Christian B, Wolf-Rudiger S, Dimitrios G. Effects of hypothermia on excitatory amino acids and metabolism in stroke patients: a microdialysis study. Stroke. 2002;33:519-524. doi:10.1161/hs0102. 100878

30. Clayton TA, Lindon JC, Cloarec O, Antti H, Charuel C, Hanton G Pharmaco-metabonomic phenotyping and personalized drug treatment. Nature. 2006;440:1073-1077. doi:10.1038/nature04648

31. Csermely P, Söti C, Blatch GL. Chaperones as parts of cellular networks. Adv Exp Med Biol. 2007;594:55-63.
Drug Design, Development and Therapy

\section{Publish your work in this journal}

Drug Design, Development and Therapy is an international, peerreviewed open-access journal that spans the spectrum of drug design and development through to clinical applications. Clinical outcomes, patient safety, and programs for the development and effective, safe, and sustained use of medicines are a feature of the journal, which has also been accepted for indexing on PubMed Central. The manuscript management system is completely online and includes a very quick and fair peer-review system, which is all easy to use. Visit http://www. dovepress.com/testimonials.php to read real quotes from published authors. 